\title{
Research Article \\ Study of Dust-Acoustic Multisoliton Interactions in Strongly Coupled Dusty Plasmas
}

\author{
Najah Kabalan, Mahmoud Ahmad, and Ali Asad \\ Department of Physics, Faculty of Science, Tishreen University, Latakia, Syria \\ Correspondence should be addressed to Ali Asad; ali760633@gmail.com
}

Received 4 August 2020; Revised 12 October 2020; Accepted 2 November 2020; Published 16 November 2020

Academic Editor: Zhi-Yuan Sun

Copyright ( 2020 Najah Kabalan et al. This is an open access article distributed under the Creative Commons Attribution License, which permits unrestricted use, distribution, and reproduction in any medium, provided the original work is properly cited.

\begin{abstract}
The effect of the structure parameter on the compressibility of dust grains and soliton behavior in a dusty plasma system consisting of Maxwellian electrons, ions, and dust grains charged with a negative charge has been studied. In the theoretical study, a reductive perturbation technique was used to derive the Korteweg-de Vries (KdV) equation and employ the Hirota bilinear method to obtain multisoliton solution. It is found that coupling and structure parameters have a clear effect on the compressibility. These changes in the compressibility affected the amplitude and width of interactive solitons, in addition to the phase shifts resulting from the interaction. These results can be used to understand the behavior of solitary waves that occur in various natural and laboratory plasma environments with dust impurity situations.
\end{abstract}

\section{Introduction}

Study of nonlinear phenomena in dusty plasma had a great deal of interest because of the presence of dust in various space and astrophysical environments, for example, planetary rings, comets, the Earth's ionosphere, and interstellar molecular clouds $[1,2]$. Moreover, dusty plasma opened up a new field of research after the possibility of crystallization (whose temperature is low relative to the temperature of other components) in a crystalline structure called a plasma crystal or coulomb crystal was discovered $[3,4]$.

Dusty plasma is composed of ordinary plasma (electronion plasma) and additional solid grains whose radius range from $100 \mathrm{~nm}-100 \mu \mathrm{m}$. These grains are charged with a negative charge because the thermal velocity of electrons is greater than that of ions [2].

The Coulomb coupling parameter is one of the basic properties of the dusty plasma system, which determines the phase state of the system and is a dimensionless parameter which represents the ratio between the electrostatic interaction energy and the thermal energy of the grains, and the first investigations showed that the Coulomb coupling parameter is given as follows [5]:

$$
\Gamma_{c}=\frac{\left(e Z_{d}\right)^{2}}{4 \pi \varepsilon_{0} a T_{d}},
$$

where $a=\left(3 / 4 \pi n_{d}\right)^{1 / 3}$ interparticle distance, $T_{d}$ is the temperature of dust grains, $n_{d}$ is the grain number density, and $Z_{d}$ is the charge number of grains. Later, a new mathematical formula of the coupling parameter was obtained after considering the screening of charges, using the Debye-Hückel or Yukawa potential that is given as follows [6]:

$$
\Gamma=\frac{\left(e Z_{d}\right)^{2}}{4 \pi \varepsilon_{0} a T_{d}} \exp (-k),
$$

where $k=a / \lambda_{D}$ is the structural parameter, which represents the ratio between the interparticle distance and the Debye screening length. Vaulina and Khrapak proposed an empirical scaling law of the coupling parameter that is compatible with recent molecular dynamics simulations, which is given as follows [7]: 


$$
\Gamma=\frac{\left(e Z_{d}\right)^{2}}{4 \pi \varepsilon_{0} a T_{d}}\left(1+k+\frac{k^{2}}{2}\right)^{1 / 2} \exp (-k)
$$

The presence of dust of a relatively large mass and charge contributes to modifying the plasma collective behavior and generates new linear and nonlinear modes, for example, dust-ion-acoustic (DIA) waves [8], dust-acoustic waves (DA) [9], dust-lattice (DL) waves [10], DIA solitary waves [11], DA solitary waves [12], and DL solitary waves [13].

Many researchers have studied the interaction of nonlinear waves in dusty plasma systems using many analytical, experimental, and numerical methods. Jaiswal et al. [14] studied dust-acoustic solitary wave (DASW) head-on collision in strongly and weakly coupled dusty plasma, using the extended Poincaré-Lighthill-Kuo perturbation method. Effects of dusty plasma parameters on phase shifts of these solitary waves are studied. They found that the phase shift changes its sign when compressibility of the medium exceeds the critical value for a specific set of dusty plasma parameters. Gao et al. [15] studied the overtaking collision between two dust-acoustic waves in dusty plasmas consisting of Boltzmann electrons and ions, and negative dust grains by the PIC simulation method. They compared their results with previous theoretical studies using the Hirota method and found a significant correlation between the results of the two methods. Boruah et al. [16] experimentally investigated the propagation and interaction of dust-acoustic multisolitons which unmagnetized strongly coupled dusty plasma. By comparing their results with previous theoretical studies, they proved that the evolution of these waves depends on the amplitude of the initial perturbation. Tao et al. [17] studied the head-on collision between two dust-acoustic solitary waves in an unmagnetized strongly coupled dust plasma using the PIC simulation method. By comparing simulation results with an analytical study, they noted that the analytical results are correct if the amplitudes of both of the colliding solitary waves are sufficiently small. Seadawy and Jun [18] obtained The Zakharov-Kuznetsov-Burgers (ZKB) equations that describe the dust-ion-acoustic waves in dusty plasma with high-energy electrons and positrons by applying the modified direct algebraic method. They found that the electric field potential, electric field, and quantum statistical pressure significantly impact in the form of water wave solutions for the three-dimensional $\mathrm{ZKB}$ equation. Arnous et al. [19] applied the modified simple equation method to the complex Ginzburg-Landau equation to secure soliton solutions. They studied using this method the Kerr and power laws of nonlinearity. The results of their investigation showed that the limitation of the scheme prevents obtaining bright soliton solutions. Seadawy [20] applied the reductive perturbation procedure method on the fluid system governing plasma, and he got the nonlinear three-dimensional modified Zakharov-Kuznetsov ( $\mathrm{mZK}$ ) equation governing the propagation of ion dynamics of nonlinear ion-acoustic waves in a plasma comprising cold ions and hot isothermal electrons in the presence of a uniform magnetic field. He found that the electrostatic field potential and electric field form traveling wave solutions for the three-dimensional $\mathrm{mZK}$ equation.
The Hirota bilinear method differs from the mathematical methods used in previous investigations (for example, the extended modified direct algebraic method and extended mapping method) in that it enables us to obtain multisoliton solutions of nonlinear partial differential equations, which is why we chose this method. Also, previous studies have examined the effect of the coupling parameter on the behavior of the solitons in the strongly coupled dusty plasma but neglected the effect of the structure parameter, as this parameter is expected to play an important role. In this scenario, we relied on the results mentioned in Reference [20], which showed that the internal energy is related to the coupling parameter and the structure parameter together.

In this paper, the overtaking collision between two solitons and three solitons in strongly coupled dusty plasma is studied. The effect of the structure parameter on plasma compressibility and the behavior of the interactive solitons is taken into account. The reductive perturbation method is applied to obtain the Korteweg-de Vries (KdV) equation. The Hirota bilinear method is applied to obtain multisoliton solutions. Computer modeling used the Maple program to show the time development of the propagation and interaction of solitons.

\section{Materials and Methods}

We consider an unmagnetized strongly coupled dusty plasma system with negatively charged inertial dust grains and inertia-less electrons and the ions that can be described by Boltzmann distributions. The dust fluid equations that can describe this system are given as follows $[14,21]$ :

$$
\begin{aligned}
& \frac{\partial n_{d}}{\partial t}+\frac{\partial\left(n_{d} \vartheta_{d}\right)}{\partial x}=0, \\
& \frac{\partial \vartheta_{d}}{\partial t}+\vartheta_{d} \frac{\partial \vartheta_{d}}{\partial x}=\frac{\partial \Phi}{\partial x}-\frac{\dot{\mu}}{n_{d}} \frac{\partial n_{d}}{\partial x}, \\
& \frac{\partial^{2} \Phi}{\partial x^{2}}=\mu_{e} n_{e}+n_{d}-\mu_{i} n_{i},
\end{aligned}
$$

where $n_{d}$ is the dust grain number density, $\vartheta_{d}$ is the dust fluid velocity, $\Phi$ is the electrostatic potential, $n_{e}$ is the electron number density, and $n_{i}$ is the ion number density. The following normalization

$$
n_{d} \rightarrow \frac{n_{d}}{n_{d 0}}, \Phi \rightarrow \frac{e \varnothing}{K_{B} T_{i}}, \vartheta_{d} \rightarrow \frac{\vartheta_{d}}{C_{d}}, x \rightarrow \frac{x}{\lambda_{D}}, t \rightarrow t \omega_{p d}
$$

where $\lambda_{D}=\left(\mathrm{K}_{B} T_{i} / n_{d 0} Z_{d} e^{2}\right)^{1 / 2}$ is the dust Debye length, $C_{d}=\left(Z_{d} \mathrm{~K}_{B} T_{i} / m_{d}\right)^{1 / 2}$ is the dust-acoustic speed, $\omega_{p d}=$ $\left(n_{d 0} Z_{d}^{2} e^{2} / m_{d}\right)^{1 / 2}$ is the dust plasma frequency, and $K_{B}, n_{d 0}$, $e$, and $m_{d}$ are the Boltzmann constant, the unperturbed dust grain number density, the electron charge, and the dust grain mass, respectively. The contribution due to the compressibility $(\mu)$ in the momentum equation, equation (5), is expressed in terms of $\dot{\mu}$ where $\dot{\mu}=\mu T_{d} / Z_{d} T_{i}$ where $T_{d}, T_{i}$, and $Z_{d}$ denote the dust temperature, the ion temperature, and the 
number of electrons residing on the surface of the negatively charged dust grains, respectively. The compressibility $\mu$ is defined as [2]

$$
\mu=\frac{1}{T_{d}}\left(\frac{\partial P}{\partial n}\right)_{T_{d}}=1+\frac{u(\Gamma)}{3}+\frac{\Gamma}{9} \frac{\partial u(\Gamma)}{\partial \Gamma},
$$

where $\Gamma$ is the Coulomb coupling parameter and $u(\Gamma)$ is a measure of the excess internal energy of the system. For a weakly coupled plasma $\Gamma<1, u(\Gamma)$ can be written as $u(\Gamma) \approx-(\sqrt{3} / 2) \Gamma^{3 / 2}[22]$, while in the case of strong coupling (Yukawa fluid) $\Gamma>100$ excess internal energy can be determined as a function of $(k, \Gamma)$ by using the following relation [23]:

$$
u(k, \Gamma)=a(k) \Gamma+b(k) \Gamma^{1 / 3}+c(k)+d(k) \Gamma^{-(1 / 3)},
$$

where parameters $a(k), b(k), c(k)$, and $d(k)$ are defined as follows:

$$
\left.\begin{array}{l}
a(k)=\frac{k}{2}-0.899-0.103 k^{2}+0.003 k^{4} \\
b(k)=0.565-0.026 k^{2}-0.003 k^{4} \\
c(k)=-0.207-0.086 k^{2}+0.018 k^{4} \\
d(k)=-0.031+0.042 k^{2}-0.008 k^{4}
\end{array}\right\} .
$$

The densities of Boltzmann distributed electrons and ions at temperatures $T_{e}$ and $T_{i}$ can be written in a normalized form as

$$
\begin{aligned}
& n_{e}=\mu_{e} \exp \left(\sigma_{i} \varnothing\right), \\
& n_{i}=\mu_{i} \exp (-\varnothing),
\end{aligned}
$$

where $\sigma_{i}=T_{i} / T_{e}$ is the ratio of ion temperature and electron temperature, $\mu_{e}=1 /(\delta-1)$, and $\mu_{i}=\delta /(\delta-1)$, where $\delta$ is the ratio of equilibrium ion to electron densities.

\section{Derivation of KdV Equation}

Now, we derive the KdV equation from equations (4)-(6) by employing the reductive perturbation technique. The independent variables are stretched as

$$
\left.\begin{array}{c}
\xi=\varepsilon^{1 / 2}(x-c t), \tau=\varepsilon^{3 / 2} t \\
\frac{\partial}{\partial x}=\varepsilon^{1 / 2} \frac{\partial}{\partial \xi}, \frac{\partial}{\partial t}=-c \varepsilon^{1 / 2} \frac{\partial}{\partial \xi}+\varepsilon^{3 / 2} \frac{\partial}{\partial \tau}
\end{array}\right\}
$$

and the dependent variables are expanded as

$$
\left.\begin{array}{l}
n_{d}=1+\varepsilon n_{1}+\varepsilon^{2} n_{2}+\cdots \\
\vartheta_{d}=\varepsilon \vartheta_{1}+\varepsilon^{2} \vartheta_{2}+\cdots \\
\Phi=\varepsilon \Phi_{1}+\varepsilon^{2} \Phi_{2}+\cdots
\end{array}\right\}
$$

$\varepsilon$ is a small parameter proportional to the strength of the perturbation. Substituting (12)-(13) into (4)-(6) and taking the terms in different powers of $\varepsilon$, we obtain in the lowest order of $\varepsilon$ :

$$
\begin{aligned}
& n_{1}=-\left(\mu_{e} \sigma_{i}+\mu_{i}\right) \Phi_{1}, \\
& \vartheta_{1}=-c\left(\mu_{e} \sigma_{i}+\mu_{i}\right) \Phi_{1}
\end{aligned}
$$

where $c$ the phase velocity given as follows:

$$
c=\sqrt{\frac{1+\dot{\mu}\left(\mu_{e} \sigma_{i}+\mu_{i}\right)}{\left(\mu_{e} \sigma_{i}+\mu_{i}\right)} .}
$$

Similarly, we get from the terms of order $\varepsilon^{2}$ and $\varepsilon^{5 / 2}$ :

$$
\begin{aligned}
& -c \frac{\partial n_{2}}{\partial \xi}+\frac{\partial n_{1}}{\partial \tau}+n_{1} \frac{\partial \vartheta_{1}}{\partial \xi}+\frac{\partial \vartheta_{2}}{\partial \xi}+\vartheta_{1} \frac{\partial n_{1}}{\partial \xi}=0, \\
& -c \frac{\partial \vartheta_{2}}{\partial \xi}+\frac{\partial \vartheta_{1}}{\partial \tau}+\vartheta_{1} \frac{\partial \vartheta_{1}}{\partial \xi}=\frac{\partial \Phi_{2}}{\partial \xi}+\mu n_{1} \frac{\partial n_{1}}{\partial \xi}-\mu \frac{\partial n_{2}}{\partial \xi}, \\
& \frac{\partial^{2} \Phi_{1}}{\partial \xi^{2}}=n_{2}+\left(\mu_{e} \sigma_{i}+\mu_{i}\right) \Phi_{2}+\left(\frac{\mu_{e} \sigma_{i}^{2}-\mu_{i}}{2}\right) \varnothing_{1}^{2} .
\end{aligned}
$$

By common solution to system of equations (15)-(17), we obtain the following Korteweg-de Vries (KdV) equation for the first-order perturbed electrostatic potential $\Phi_{1}$ as follows:

$$
\frac{\partial \Phi_{1}}{\partial \tau}+A \Phi_{1} \frac{\partial \Phi_{1}}{\partial \xi}+B \frac{\partial^{3} \Phi_{1}}{\partial \xi^{3}}=0
$$

where the nonlinear coefficient $A$ and the dispersion coefficient $B$ are given by

$$
A=-\frac{1}{E}\left\{\left[\left(\mu_{e} \sigma_{i}+\mu_{i}\right)^{2}\left[3+2 \mu\left(\mu_{e} \sigma_{i}+\mu_{i}\right)\right]+\left(\mu_{e} \sigma_{i}^{2}-\mu_{i}\right)\right]\right\},
$$

$$
B=\frac{1}{E}
$$

where

$$
E=\left\{\frac{\left(\mu_{e} \sigma_{i}+\mu_{i}\right)}{c}\left[1+2 \dot{\mu}\left(\mu_{e} \sigma_{i}+\mu_{i}\right)\right]\right\} .
$$

\section{Multisoliton Solutions}

For obtaining the multisoliton solution of equation (16) and to study the interaction between them, to do so, we shall employ the Hirota bilinear method [23].

The first step: using the transformation on equation (19):

$$
\Phi_{1}=\frac{12 B}{A} \frac{\partial^{2}(\ln (f(\xi, \tau)))}{\partial \xi^{2}}
$$


We get the following equation:

$$
-f_{\xi} f_{\tau}+f f_{\xi \tau}+B f f_{\xi \xi \xi \xi}-4 B f_{\xi \xi \xi} \cdot f_{\xi}+3 B\left(f_{\xi \xi}\right)^{2}=0 .
$$

By using the Hirota-D operator, we get

$$
\begin{aligned}
& D_{\tau} D_{\xi}\{f, f\}=2\left(f f_{\xi \tau}-f_{\xi} f_{\tau}\right), \\
& B D_{\xi}^{4}\{f, f\}=2\left(B f f_{\xi \xi \xi \xi}-4 \mathrm{~B} f_{\xi} f_{\xi \xi \xi}+3 B\left(f_{\xi \xi}\right)^{2}\right) .
\end{aligned}
$$
form:

Using (25) and (26) in (24), we get the Hirota bilinear

$$
\left(D_{\tau} D_{\xi}+B D_{\xi}^{4}\right)\{f, f\}=0
$$

where $D$ is a binary operator (because it operates on a pair of functions) and is called the Hirota derivative.

We use Hirota's perturbation technique, and we insert $f=1+\varepsilon f_{1}$ where $f_{1}=e^{\theta_{1}}$; substituting $\varepsilon=1$, we obtain $f=1$ $+f_{1}$; now substituting in equation (23), we get the singlesoliton solution as follows:

$$
\Phi_{1}=\frac{12 B}{A} \frac{\partial^{2}\left\{\ln \left[1+e^{\left(k_{1} B^{-(1 / 3)} \xi-k_{1}^{3} \tau\right)}\right]\right\}}{\partial \xi^{2}} .
$$

Same as the previous method, we insert $f=1+\varepsilon f_{1}+\varepsilon^{2} f_{2}$ where $f_{1}=e^{\theta_{1}}+e^{\theta_{2}}$ and make some necessary mathematical calculations. We get the relationship $f_{2}=a(1,2) e^{\theta_{1}+\theta_{2}}$; now substituting in equation (23), we get the two-soliton solution as follows:

$$
\Phi_{1}=\frac{12 B}{A} \frac{\partial^{2}\left\{\ln \left[1+e^{\theta_{1}}+e^{\theta_{1}}+a(1,2) e^{\theta_{1}+\theta_{2}}\right]\right\}}{\partial \xi^{2}},
$$

where $\theta_{i}=k_{i} B^{-(1 / 3)} \xi-k_{i}^{3} \tau-\Delta_{i}, i=1,2, \Delta_{i}=\mp\left(2 B^{1 / 3} / k_{i}\right) \ln \mid$ $\sqrt{a(1,2)} \mid$ are the phase shifts $a(1,2)=\left(k_{1}-k_{2}\right)^{2} /\left(k_{1}+k_{2}\right)^{2}$ where $k_{1}, k_{2}$ are wave numbers.

In order to get a three-soliton solution, we insert $f=1+\varepsilon f_{1}+\varepsilon^{2} f_{2}+\varepsilon^{3} f_{3}$ twhere $f_{1}=e^{\theta_{1}}+e^{\theta_{2}}+e^{\theta_{3}} \cdot f_{2}$ and $f_{3}$ are determined by performing some mathematical calculations, and we get the following two relationships:

$$
\begin{aligned}
& f_{2}=a(1,2) e^{\theta_{1}+\theta_{2}}+a(1,3) e^{\theta_{1}+\theta_{3}}+a(2,3) e^{\theta_{2}+\theta_{3}}, \\
& f_{3}=b e^{\theta_{1}+\theta_{2}+\theta_{3}} .
\end{aligned}
$$

Substituting in equation (23), we get the two-soliton solution as follows:

$$
\begin{aligned}
\Phi_{1}= & \frac{12 B}{A} \frac{\partial^{2}}{\partial \xi^{2}}\left\{\operatorname { l n } \left[1+e^{\theta_{1}}+e^{\theta_{2}}+e^{\theta_{3}}+a(1,2) e^{\theta_{1}+\theta_{2}}\right.\right. \\
& \left.\left.+a(1,3) e^{\theta_{1}+\theta_{3}}+a(2,3) e^{\theta_{2}+\theta_{3}}+b e^{\theta_{1}+\theta_{2}+\theta_{3}}\right]\right\},
\end{aligned}
$$

where $\theta_{1}=k_{1} B^{-(1 / 3)} \xi-k_{1}^{3} \tau-\Delta_{1}^{\prime}, \quad \theta_{2}=k_{2} B^{-(1 / 3)} \xi-k_{2}^{3} \tau-\Delta_{2}^{\prime}$, and $\theta_{3}=k_{3} B^{-(1 / 3)} \xi-k_{3}^{3} \tau-\Delta_{3}^{\prime}$.

The phase shifts are given as follows:

$$
\begin{aligned}
\Delta_{1}^{\prime} & =\mp \frac{2 B^{1 / 3}}{k_{1}} \ln \left|\frac{b}{a(2,3)}\right|, \quad \Delta_{2}^{\prime}=\mp \frac{2 B^{1 / 3}}{k_{2}} \ln \left|\frac{b}{a(1,3)}\right|, \quad \Delta_{3}^{\prime} \\
& =\mp \frac{2 B^{1 / 3}}{k_{3}} \ln \left|\frac{b}{a(1,2)}\right|,
\end{aligned}
$$

where $a(1,2)=\left(k_{1}-k_{2}\right)^{2} /\left(k_{1}+k_{2}\right)^{2}, \quad a(1,3)=\left(k_{1}-k_{3}\right)^{2} /$ $\left(k_{1}+k_{3}\right)^{2}, a(2,3)=\left(k_{2}-k_{3}\right)^{2} /\left(k_{2}+k_{3}\right)^{2}$, and $b=a(1,2) a(1$, 3) $a(2,3)$.

\section{Results and Discussion}

In this work, we investigated the propagation and interaction of DA multisolitons in strongly coupled dusty plasma consisting of Maxwellian electrons, ions, and inertial negative dust grains. The Korteweg-de Vries (KdV) equation (19) was obtained using a reductive perturbation technique. Soliton solutions are formed due to the balance between the nonlinear coefficient $A$ and the dispersion coefficient $B$. It is important to indicate the numerical data used in this study obtained from References [24, 25]. The range of structure parameter and the coupling parameter values were chosen based on experimental data, which describes the Yukawa fluid in the case of strong coupling, taken from References [26, 27].

5.1. Compressibility Changes. We plotted the compressibility variations with the coupling parameter for different values of the structure parameter and obtained Figure 1.

In Figure 1, it is shown that the compressibility decreases with the increasing coupling parameter, while the compressibility increases with the increase in the structure parameter value. The increase in the structure parameter means an increase of interparticle distance and a decrease of the Debye screening length; hence, the grains would become more mobile to move. This causes an increase of its compressibility. We compare this result with the result presented in Figure 2 from Reference [17].

In Figure 2, the change of compressibility when the plasma is transferred from the weak coupling state $(\Gamma<1)$ to a strong coupling state $(\Gamma>1)$ while neglecting the effect of the structure parameter $(k=0)$ is shown. By comparing the two shapes (1) and (2), it appears that there is a great agreement between our results and the results of the aforementioned reference when the plasma becomes a strong coupling state where the compressibility values become negative.

5.2. Soliton Shape Changes. We plotted the single-soliton shape relation (28) variations for different values of the structure parameter and obtained Figure 3.

We plotted the single-soliton shape relation (25) variations for different values of the coupling parameter and obtained Figure 4. 


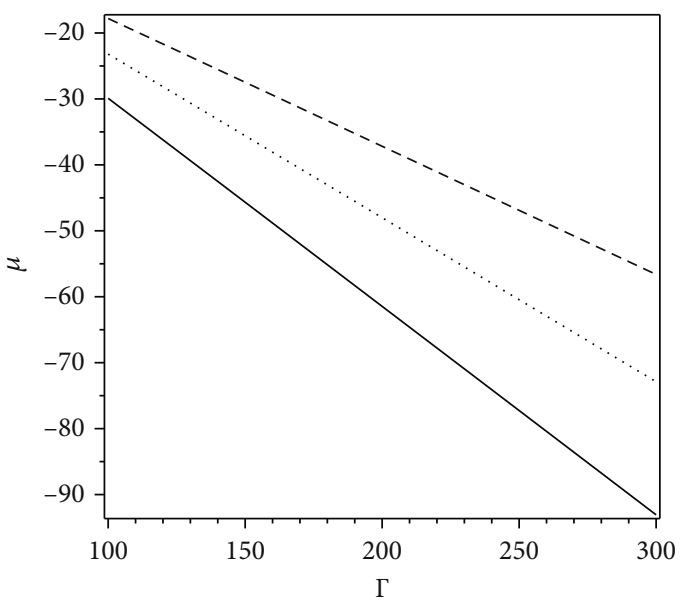

FIGURE 1: Compressibility variations with the coupling parameter for different values of the structure parameter, $k=0.4$ (solid line), $k=0.8$ (dot line), and $k=1.2$ (dashed line).

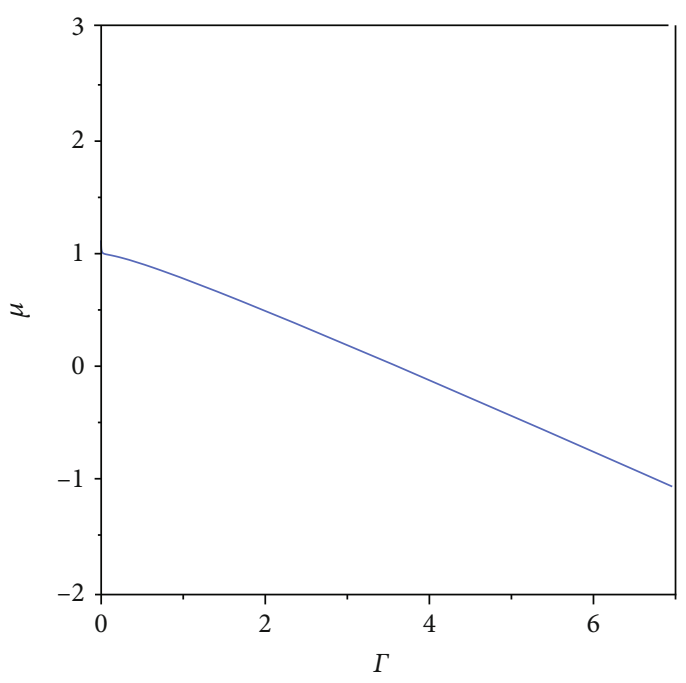

FIgURE 2: Compressibility variations with the coupling parameter from Reference [17].

Figure 3 shows that the amplitude and width of the soliton increases with the increase of the structure parameter (compressibility increasing) for a constant value of the coupling parameter $\Gamma=170$. Figure 4 shows that the amplitude and width of the soliton decreases with the increase of the coupling parameter (compressibility decreases) for a constant value of the structure parameter $k=0.8$. It is observed from Figure 3 that the amplitude of the soliton decreases significantly when the value of the coupling parameter increases from $\Gamma=100$ (dashed line) to $\Gamma=170$ (dotted line). It is the critical value of the plasma transmission from the fluid phase to a quasisolid structure called the plasma crystal. These results correspond with the simulation results in Reference [17] and with the theoretical study that did not take into account the effect of the structure parameter on the compressibility (i.e., $k=0$ ) in Reference [14].

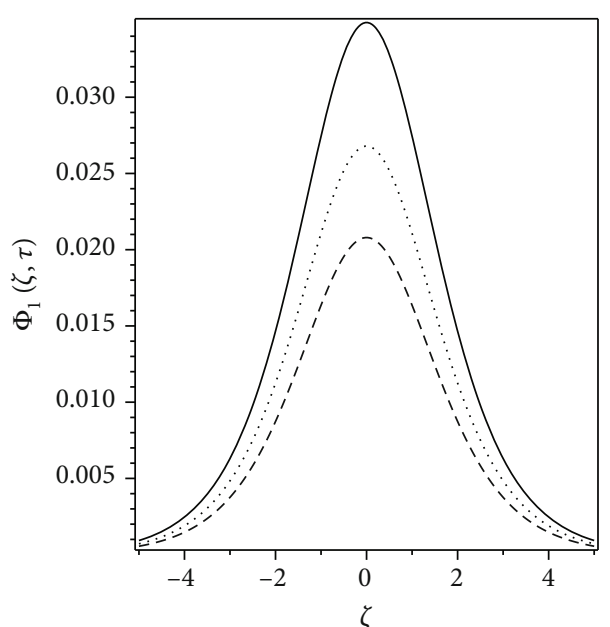

FIGURE 3: Single-soliton shape variations for different values of the structure parameter, $k=1.2$ (solid line), $k=0.8$ (dot line), and $k=0.4$ (dashed line). $\sigma=0.1, \delta=10$, and $\Gamma=170$.

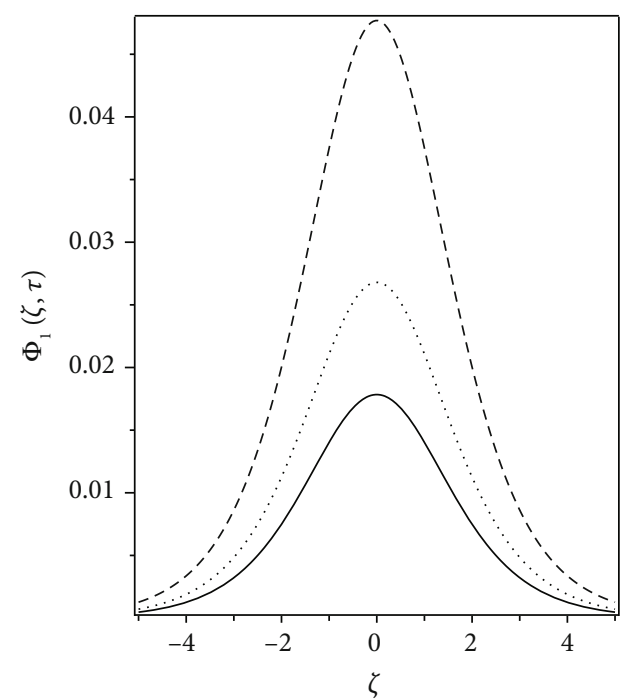

FIGURE 4: Single-soliton shape variations for different values of the coupling parameter, $\Gamma=250$ (solid line), $\Gamma=170$ (dot line), and $\Gamma=100$ (dashed line). $\sigma=0.1, \delta=10$, and $k=0.8$.

5.3. Time Evolution of Multisolitons. The mathematical study used in this investigation differs from the previous investigations $[18,19,28]$, as they used the Zakharov-Kuznetsov equation $(\mathrm{ZK})$, which is an appropriate equation for studying the $(2+1)$-dimensional systems. But the reason for using the Korteweg-de Vries (KdV) equation in this investigation is that the $\mathrm{KdV}$ equation describes multisoliton solutions depending on the initial conditions, as well as the possibility of applying the Hirota direct method to this equation. Thus, this equation fulfills the required purpose of our investigation. The Hirota method is an innovative and powerful method by which we can obtain, in principle, any number of solutions for many nonlinear partial differential equations.

In this section, we used the Mable program to perform a numerical simulation showing the propagation and 

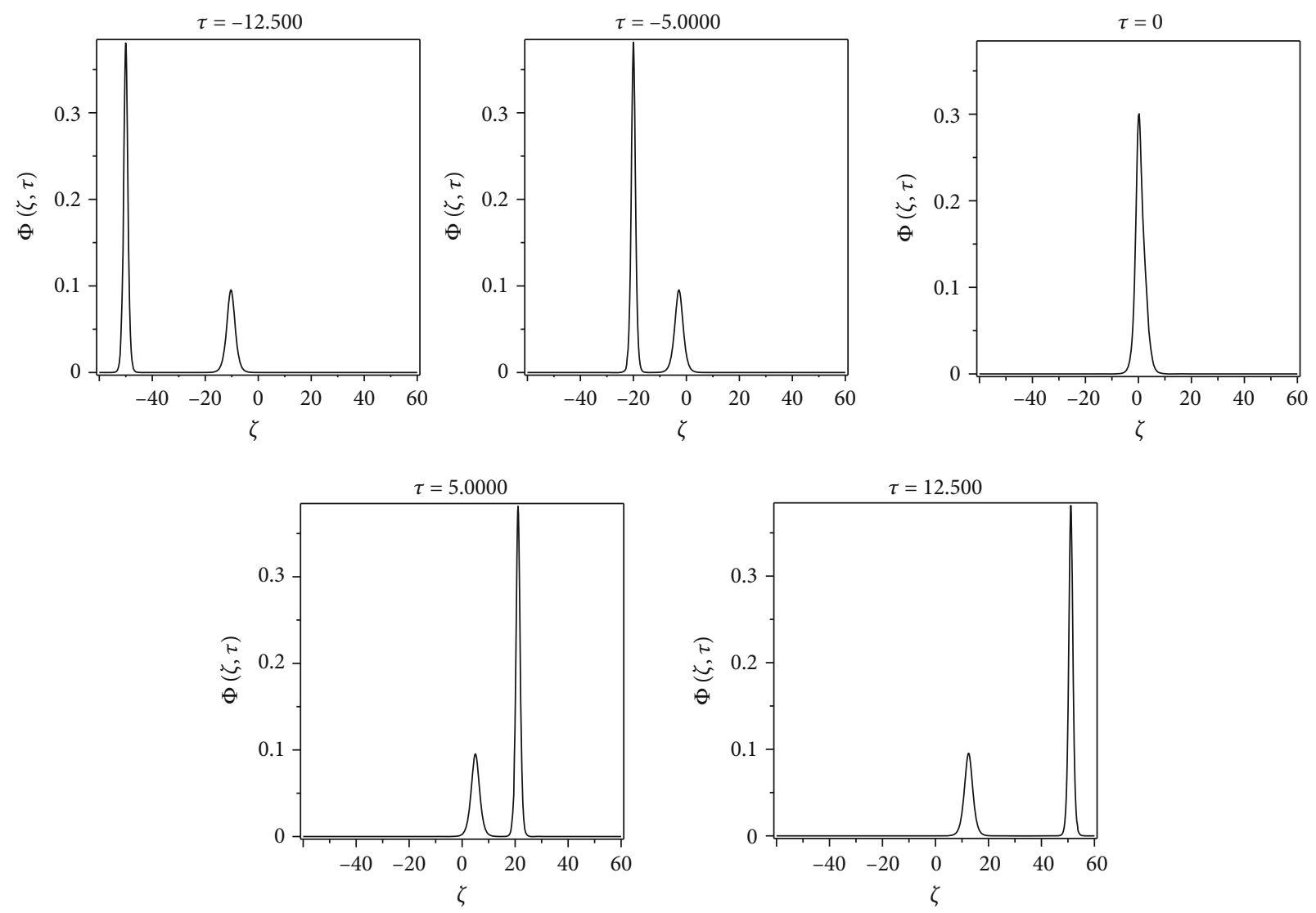

Figure 5: Time evolution of two solitons at different times, $\sigma=0.1, \delta=10, k=0.8, \Gamma=170, k_{1}=1$, and $k_{2}=2$.

interaction of solitons moving in the same direction, and we obtained the following results.

Figure 5 shows the time evolution of two solitons with different amplitudes and widths moving in the same direction from left to right at different times of $\tau$. At $\tau=-12.5$, the larger amplitude soliton is behind the smaller amplitude soliton. With the passage of time, a large soliton approaches a small soliton at $\tau=-5$ because a higher amplitude soliton travels faster than a smaller amplitude soliton. At $\tau=0$, the two solitons merge and become one soliton where the amplitude of this soliton is less than the amplitude of the large soliton and greater than the amplitude of the small soliton. At $\tau=5$, they separate from each other again, and each soliton acquires its shape and speed before the reaction, but the large soliton comes in front.

Similarly, the two-soliton interaction scenario was applied on the time evolution of three solitons with different amplitudes and widths moving in the same direction from left to right at different times of $\tau$; the results are shown in Figure 6. It should be noted that the results of simulation of the time evolution of propagation and interaction of solitons in this work correspond with the experimental results in Reference [16].

5.4. Phase Shifts. In this section, we studied the effect of the structure parameter on the phase shifts of interaction solitons, and we obtained the following results.
Figures 7 and 8 show a decrease of phase shift as the structure parameter increases. The reason for the decrease in the phase shift value is due to the phase shift being related to the dispersion coefficient $B$, which in turn is related to the structure parameter as shown in relation (21). In other words, the phase shifts of solitons after interaction increase as the coupling strength between dust particles increases. This is achieved when the value of the structure parameter is small, that is, the interparticle distance becomes smaller. When we neglect the effect of the structure parameter and study the phase shift changes with compressibility, we get results similar to the theoretical study and PIC simulation in the Figure 5 of Reference [18]. The difference between our study and their study is in the method used, as they used the Poincaré-Lighthill-Kuo (PLK) method in a study of the head-on collision between two dust-acoustic solitary waves. Any waves moving in opposite directions, in which case the effect of the collision is greater, cause an increase in the phase shifts resulting from the collision. In addition, phase shifts are decreasing with the wave number increasing. This corresponds with the results in Reference [29].

\section{Conclusions}

In this work, the effect of the structure parameter on the compressibility of dust grains and soliton behavior in a dusty plasma system consisting of Maxwellian electrons, ions, and 

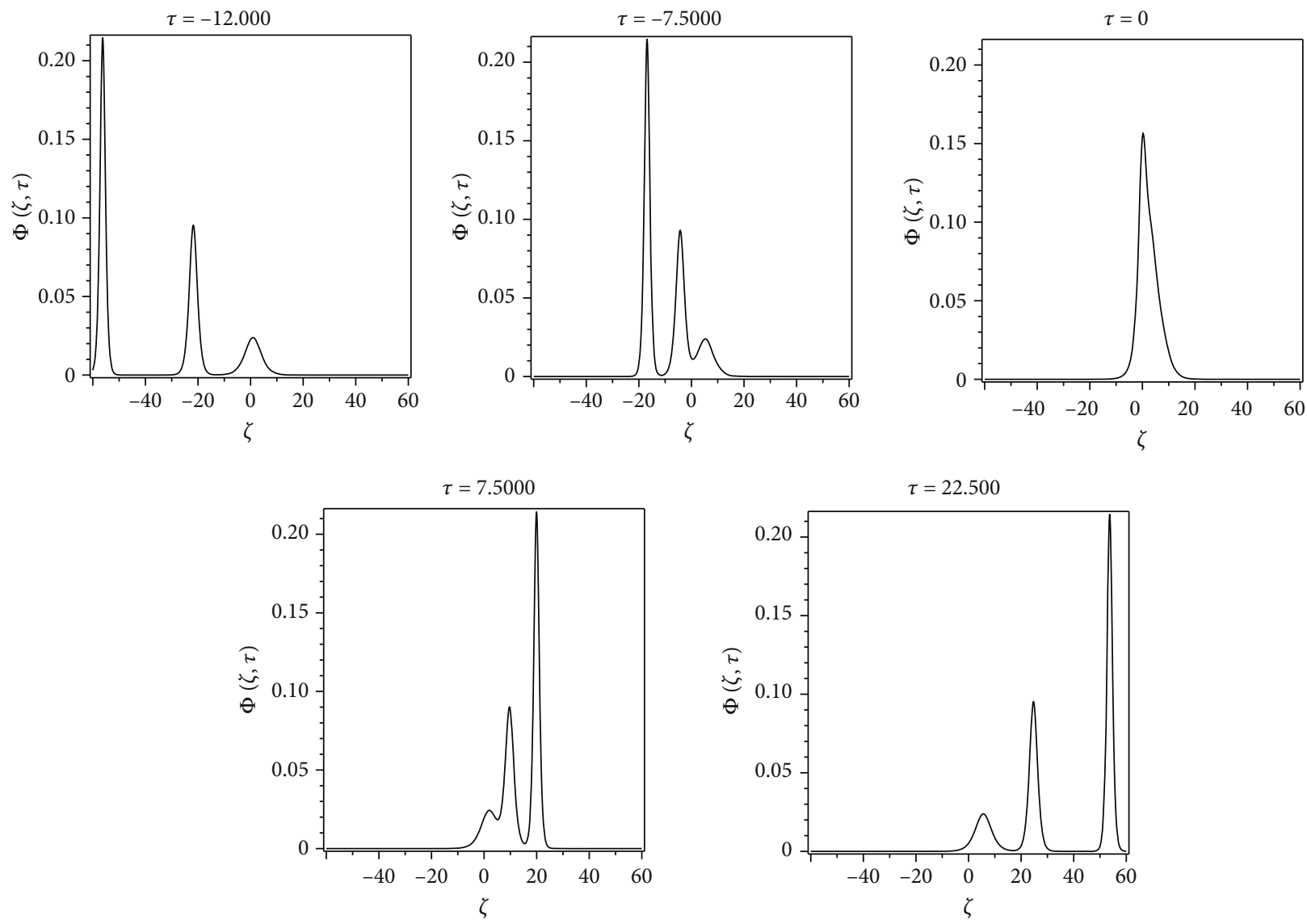

Figure 6: Time evolution of three solitons at different times, $\sigma=0.1, \delta=10, k=0.8, \Gamma=170, k_{1}=0.5, k_{2}=1$, and $k_{3}=1.5$.

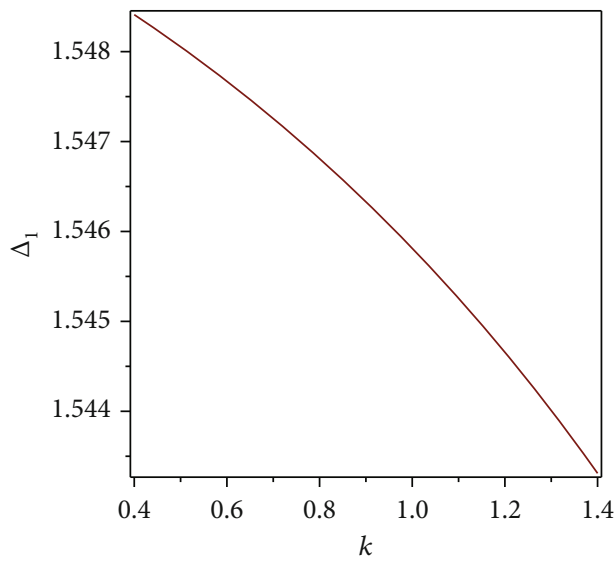

(a)

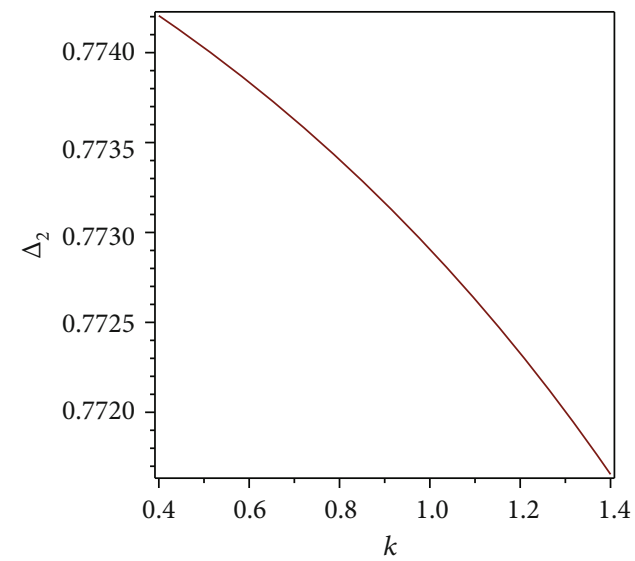

(b)

FIGURE 7: Variation of the phase shift for two solitons against the structure parameter, for small soliton $k_{1}=1$ (a) and for large soliton $k_{2}=2$ (b), for fixed values of $\sigma=0.1, \delta=10$, and $\Gamma=170$.

dust grains charged with a negative charge has been studied. In the theoretical study, a reductive perturbation technique was used to derive the Korteweg-de Vries (KdV) equation, employing the Hirota bilinear method for obtaining a multisoliton solution. The Mable program was used to perform a numerical simulation showing the propagation and interac- tion of solitons. The results obtained can be summarized as follows:

(i) Coupling and structure parameters have a clear effect on the compressibility, where the compressibility increases with the increase of the structural 


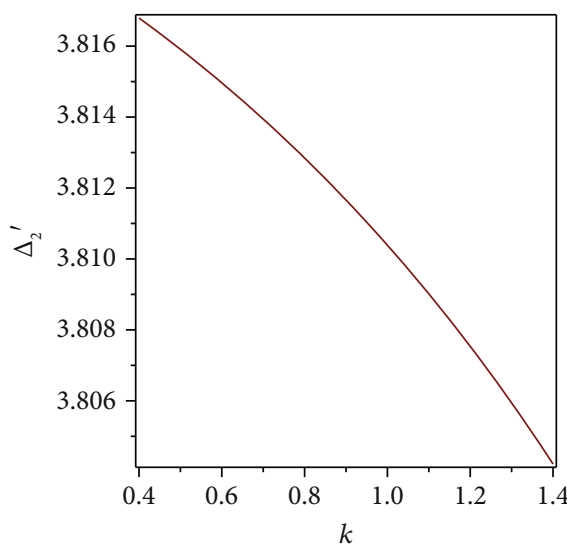

(a)

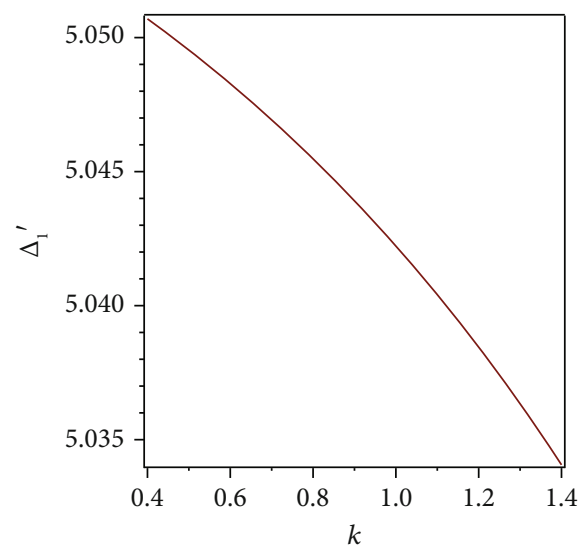

(b)

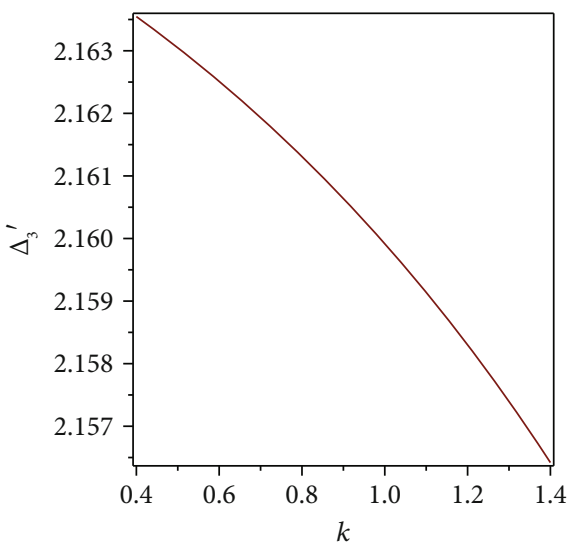

(c)

FIGURE 8: Variation of the phase shift for three solitons against the structure parameter, for the small soliton $k_{1}=0.5$ (a), for the medium soliton $k_{2}=1(\mathrm{~b})$, and for the large soliton $k_{3}=1.5(\mathrm{c})$, for fixed values of $\sigma=0.1, \delta=10$, and $\Gamma=170$.

parameter and decreases with increase of the coupling parameter

(ii) Compressibility changes contributed to modifying the shape of the soliton, where amplitude and width of the soliton increases with the increase of the structure parameter and decreases with the increase of the coupling parameter

(iii) Numerical simulation of propagation and interaction of solitons showed interest corresponding with previous relevant theoretical and experimental works

(iv) The phase shift of the interactive solitons decreases with structure parameter and wave number increase

Our results showed that the smaller the distance between the grains, the more strongly coupled the dusty plasma, so the structure parameter plays an important role in determining the phase state of the dusty plasma, which clearly affects the behavior of the nonlinear dusty acoustic pattern propagation in this type of plasma.

Analytical and numerical solutions of nonlinear partial differential equations are useful in enabling us to deeply understand the behavior of nonlinear phenomena in complex plasma systems. Moreover, solitons have played a very important and useful role in communication, where optical soliton pulses contributed running over long distances and transmitting high data rate information in optical fiber [30]. In biology, soliton theory has been used to describe signal and energy propagation in biomembranes as occurs, for example, in the nervous system and to low frequency collective pattern proteins and DNA [31].

\section{Data Availability}

No data were used to support this study.

\section{Conflicts of Interest}

The authors declare that there are no conflicts of interest regarding the publication of this paper.

\section{Acknowledgments}

This study was supported by Tishreen University. 


\section{References}

[1] R. Bingham, U. De Angelis, V. N. Tsytovich, and O. Havnes, "Electromagnetic wave scattering in dusty plasmas," Physics of Fluids B: Plasma Physics, vol. 3, no. 3, pp. 811-817, 1991.

[2] P. K. Shukla and A. A. Mamun, Introduction to Dusty Plasma Physics, Institute of Physics, Bristol, UK, 2002.

[3] A. Barkan, R. L. Merlino, and N. D'Angelo, "Laboratory observation of the dust-acoustic wave mode," Physics of Plasmas, vol. 2, no. 10, pp. 3563-3565, 1995.

[4] R. L. Merlino, A. Barkan, C. Thompson, and N. D’Angelo, "Laboratory studies of waves and instabilities in dusty plasmas," Physics of Plasmas, vol. 5, no. 5, pp. 1607-1614, 1998.

[5] E. L. Pollock and J. P. Hansen, "Statistical mechanics of dense ionized matter. II. Equilibrium properties and melting transition of the crystallized one-component plasma," Physical Review, vol. 8, no. 6, pp. 3110-3122, 1973.

[6] H. Ikezi, "Coulomb solid of small particles in plasmas," Physics of Fluids, vol. 29, no. 6, p. 1764, 1986.

[7] O. S. Vaulina and S. A. Khrapak, "Scaling law for the fluidsolid phase transition in Yukawa systems (dusty plasmas)," Journal of Experimental and Theoretical Physics, vol. 90, no. 2, pp. 287-289, 2000.

[8] P. K. Shukla and V. P. Silin, "Dust ion-acoustic wave," Physica Scripta, vol. 45, no. 5, pp. 508-509, 1992.

[9] N. N. Rao, P. K. Shukla, and M. Y. Yu, "Dust-acoustic waves in dusty plasmas," Planetary and Space Science, vol. 38, no. 4, pp. 543-546, 1990.

[10] F. Melands, "Lattice waves in dust plasma crystals," Physics of Plasmas, vol. 3, no. 11, pp. 3890-3901, 1996.

[11] M. R. Amin, G. E. Morfill, and P. K. Shukla, "Modulational instability of dust-acoustic and dust-ion-acoustic waves," Physical Review, vol. 58, no. 5, pp. 6517-6523, 1998.

[12] A. A. Mamun and P. K. Shukla, "Linear and nonlinear dusthydromagnetic waves," Physics of Plasmas, vol. 10, no. 11, pp. 4341-4349, 2003.

[13] S. Ghosh and M. R. Gupta, "Solitary waves in two-dimensional dusty plasma crystal: effects of weak magnetic field," Physics of Plasmas, vol. 17, no. 3, article 034505, 2010.

[14] S. Jaiswal, P. Bandyopadhyay, and A. Sen, "Theoretical study of head-on collision of dust acoustic solitary waves in a strongly coupled complex plasma," Physics of Plasmas, vol. 21, no. 5, article 053701, 2014.

[15] D. N. Gao, H. Zhang, J. Zhang, Z. Z. Li, and W. S. Duan, "Numerical modelling of overtaking collisions of dust acoustic waves in plasmas," The European Physical Journal D, vol. 70, no. 11, p. 235, 2016.

[16] A. Boruah, S. K. Sharma, Y. Nakamura, and H. Bailung, "Observation of dust acoustic multi-solitons in a strongly coupled dusty plasma," Physics of Plasmas, vol. 23, no. 9, article 093704, 2016.

[17] L. L. Tao, F. P. Wang, D. N. Gao, H. Zhang, and W. S. Duan, "Effect of the pressure of the dust grains in strongly coupled dusty plasma on the head-on collision between two nonlinear waves," Journal of Plasma Physics, vol. 86, no. 1, 2020.

[18] Abdullah, A. R. Seadawy, and W. Jun, "Mathematical methods and solitary wave solutions of three-dimensional ZakharovKuznetsov-Burgers equation in dusty plasma and its applications," Results in Physics, vol. 7, pp. 4269-4277, 2017.
[19] A. H. Arnous, A. R. Seadawy, R. T. Alqahtani, and A. Biswas, "Optical solitons with complex Ginzburg-Landau equation by modified simple equation method," Optik, vol. 144, pp. 475-480, 2017.

[20] M. Rosenberg and G. Kalman, "Dust acoustic waves in strongly coupled dusty plasmas," Physical Review E, vol. 56, no. 6, pp. 7166-7173, 1997.

[21] H. F. Darweesh, A. M. El-Hanbaly, and E. M. Abulwafa, "Theoretical study of dust acoustic solitary waves interaction in a strongly coupled dusty plasma with nonextensive electrons and ions," Quantum Physics Letters, vol. 7, no. 1, pp. 21-28, 2018.

[22] S. Ichimaru, H. Iyetomi, and S. Tanaka, "Statistical physics of dense plasmas: thermodynamics, transport coefficients and dynamic correlations," Physics Reports, vol. 149, no. 2-3, pp. 91-205, 1987.

[23] R. Hirota, The Direct Method in the Soliton Theory, Cambridge University Press, UK, 2004.

[24] U. Klein and J. Kerp, Physics of the Interstellar Medium, Argelander Institute for Astronomy, 1st edition, 2008.

[25] P. Bandyopadhyay, G. Prasad, A. Sen, and P. K. Kaw, "Experimental study of nonlinear dust acoustic solitary waves in a dusty plasma," Physical Review Letters, vol. 101, no. 6, 2008.

[26] Z. Donko, P. Hartmann, and J. Goree, "Shear viscosity of strongly-coupled two-dimensional Yukawa liquids: experiment and modeling," Modern Physics Letters B, vol. 21, no. 21, pp. 1357-1376, 2011.

[27] V. Nosenko, J. Goree, and A. Piel, "Cutoff wave number for shear waves in a two-dimensional Yukawa system (dusty plasma)," Physical Review Letters, vol. 97, no. 11, 2006.

[28] A. R. Seadawy, "Three-dimensional nonlinear modified Zakharov-Kuznetsov equation of ion-acoustic waves in a magnetized plasma," Computers \& Mathematics with Applications, vol. 71, no. 1, pp. 201-212, 2016.

[29] K. Roy, S. K. Ghosh, and P. Chatterjee, "Two-soliton and three-soliton interactions of electron acoustic waves in quantum plasma," Pramana, vol. 86, no. 4, pp. 873-883, 2016.

[30] R. Sharma and G. P. Singh, "Optical soliton \& applications thereof," International Journal of Electronics, vol. 4, no. 4, pp. 71-76, 2014.

[31] J. H. Geesink and D. K. F. Meijer, "Bio-soliton model that predicts non-thermal electromagnetic radiation frequency bands, that either stabilize or destabilize life conditions," General Physics, vol. 36, no. 4, pp. 357-378, 2016. 